\title{
A Monte Carlo radiative transfer model of satellite waveform lidar
}

\author{
Peter North ${ }^{1}$, Jacqueline Rosette ${ }^{2}$, Juan Suarez ${ }^{3}$ and Sietse Los ${ }^{4}$ \\ ${ }^{1}$ Climate and Land-Surface Systems Interaction Centre, Swansea University, SA2 8PP, \\ U.K.p.r.j.north@swansea.ac.uk \\ ${ }^{2}$ Climate and Land-Surface Systems Interaction Centre, Swansea University, SA2 8PP, \\ UK.ggrosette@swansea.ac.uk \\ ${ }^{3}$ Research Agency of the Forestry Commission, Northern Research Station, Roslin, \\ Midlothian, EH25 9SY, UK. juan.suarez@forestry.gsi.gov.uk \\ ${ }^{4}$ Climate and Land-Surface Systems Interaction Centre, Swansea University, SA2 8PP, \\ U.K. s.o.los@swansea.ac.uk
}

\begin{abstract}
We present a method and initial results for a model of the interaction of waveform lidar with a three-dimensional canopy representation. The model is developed from the FLIGHT radiative transfer model (North, 1996), based on Monte Carlo simulation of photon transport. Foliage is represented by structural properties of leaf area, leaf angle distribution (LAD), crown dimensions and fractional cover, and the optical properties of leaves, branch, shoot and ground components. Important characteristics of the model are that it can represent multiple scattering of light within the canopy and with the ground surface, simulate the return signal efficiently at multiple wavebands, and model the effects of topography. Spatial and temporal sampling characteristics of the lidar instrument are explicitly modelled. A sensitivity analysis gives expected effects of canopy parameters on the waveform, and indicates potential for retrieval of the canopy properties of fractional cover and leaf area, in addition to height.
\end{abstract}

Keywords: Waveform lidar, ICESat GLAS, radiative transfer model

\section{Introduction}

Global datasets of land surface biophysical variables are required from remote sensing to drive land surface parameterisations coupled to atmospheric general circulation models, and to calculate the exchange of carbon, water, energy and momentum fluxes between the land and atmosphere (Sellers et al., 1996; North, 2002; Alton et al., 2007). By recording temporal return, light detection and ranging (lidar) offers a unique measurement directly related to vegetation canopy height. While hitherto mostly applied using airborne platforms at local scale, the Geoscience Laser Altimeter System (GLAS) aboard the Ice, Cloud and land Elevation Satellite (ICESat) provides an opportunity to contribute to forest quantification and monitoring at regional and global scales (Schutz et al., 2005). Previous work supports the use of this data source for the estimation of canopy height and sub-canopy terrain, and, by correlation, further properties such as biomass (Lefsky et al., 2005; Harding and Carabajal, 2005; Rosette et al., 2008a). There is also ongoing research in estimation of further parameters such as vegetation cover, stemwood volume and plant area index (PAI), and their vertical profile (Koetz et al., 2006; Rosette et al., 2008b). 


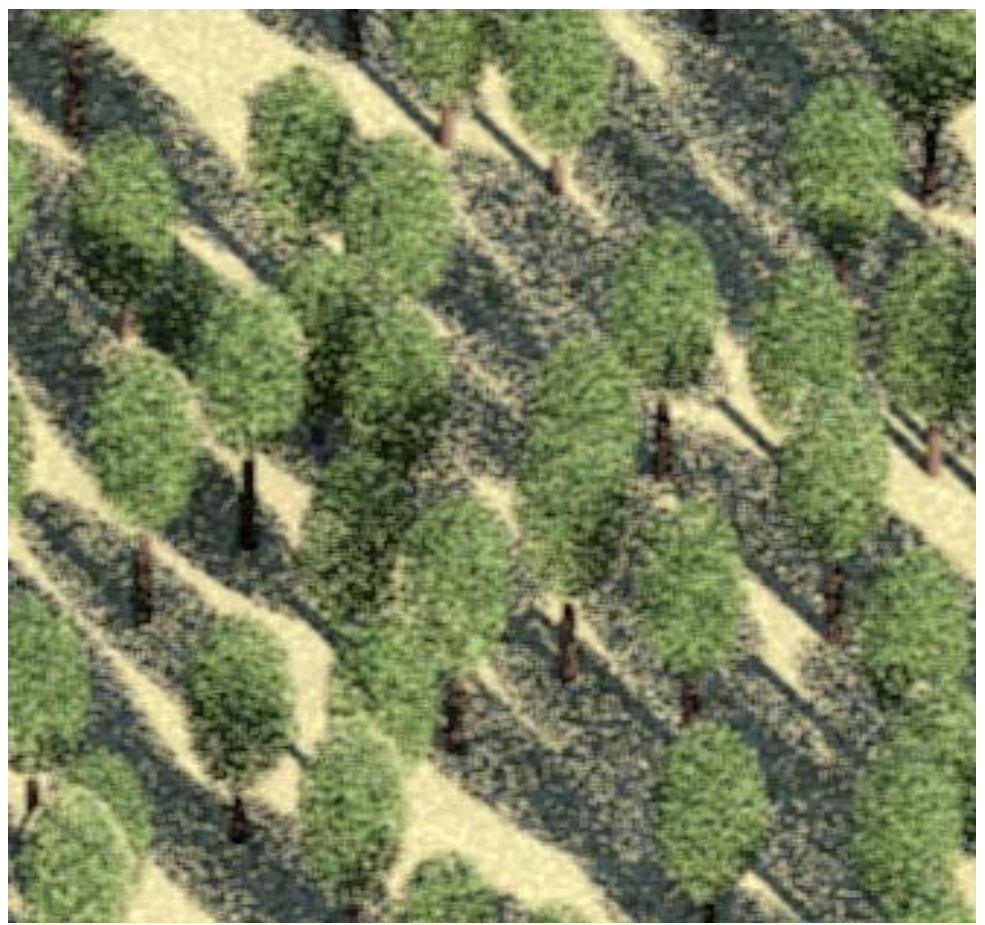

Figure 1: Example FLIGHT model output showing scene reflectance under solar illumination.

Increasingly, physically-based radiative transfer models of vegetation canopies have been used to constrain retrieval of land surface biophysical parameters, either by direct inversion or use in algorithm development. For lidar, radiative transfer models have been developed originally for atmospheric simulation (Platt, 1981), and recently several models have been developed for vegetation canopies which treat the light interaction at various degrees of complexity (Govaerts and Verstraete, 1998; Ni-Meister et al., 2001; Kotchenova et al., 2003; Disney et al., 2006).

In this work we aim to extend the three-dimensional radiative transfer model FLIGHT (North, 1996) to model waveform lidar interaction at scales suitable for ICESat interpretation. The model is based on Monte Carlo solution of radiative transfer, and offers a consistent link from lidar-derived structure to full canopy optical response and vegetation photosynthesis (Barton and North, 2001; Alton et al., 2005). A further aim is to explore the theoretical potential of biophysical parameter retrieval from satellite waveform lidar.

\section{Method}

The Method is based on Monte Carlo evaluation of photon transport. Monte Carlo simulation is a versatile technique, which allows highly accurate estimation of light interception and bidirectional reflectance (Disney, et al., 2000). The technique requires sampling of the photon free-path within a canopy representation, and simulation of the scattering event at each interaction. By iteration we obtain accurate treatment of light interception and multiple scattering between foliage elements and the soil boundary. Overlapping crowns, and multiple scattering within and between different crowns and the ground surface are thus modelled. The particular challenge of modelling lidar interaction is the additional inclusion of time dependency of the response, governed by varying path length over multiple interactions forming the return, and the temporal spread of the incoming pulse. 
Table 1: FLIGHT canopy input parameters

\begin{tabular}{cccc}
\hline Name & Units & Meaning & Value \\
\hline PAI & $\mathrm{m} 2 / \mathrm{m} 2$ & Plant area index (one sided) & 3.0 \\
LAD & - & Leaf angle distribution & Spherical \\
$\mathrm{F}_{\mathrm{c}}$ & - & Crown fractional coverage & 0.7 \\
$\mathrm{~F}_{\mathrm{g}}$ & - & Fraction of green foliage & 0.8 \\
$\mathrm{~F}_{\mathrm{b}}$ & - & Fraction of bark & 0.2 \\
$\mathrm{E}_{\mathrm{xy}}$ & $\mathrm{m}$ & Ellipsoid horizontal radius & 8.0 \\
$\mathrm{E}_{\mathrm{z}}$ & $\mathrm{m}$ & Ellipsoid vertical eccentricity & 8.0 \\
$\mathrm{D}_{\mathrm{l}}$ & $\mathrm{m}$ & Leaf size diameter & 0.05 \\
$\mathrm{DBH}$ & $\mathrm{m}$ & Trunk diameter at breast & 0.1 \\
$\mathrm{~S}_{\mathrm{r}}$ & $0-1$ & height & 0 \\
$\mathrm{~S}_{\mathrm{y}}$ & $\mathrm{Deg}$ & Soil roughness & 0 \\
$\mathrm{H}_{\min }, \mathrm{H}_{\max }$ & $\mathrm{m}$ & Tin/max height to crown & 15,20 \\
$\rho_{\mathrm{L}}$ & - & start & 0.4 \\
$\tau_{\mathrm{L}}$ & - & Leaf reflectance & 0.4 \\
$\rho_{\mathrm{S}}$ & - & Leaf transmittance & 0.15 \\
& & Soil reflectance & \\
\hline
\end{tabular}

\subsection{Canopy representation}

Foliage is approximated by structural parameters of area density, angular distribution, and size, and optical properties of reflectance and transmittance. The foliage is constrained to lie within geometric envelopes, defined by ellipsoidal or conical primitives. The locations of the crowns are normally generated statistically, parameterized by crown fractional cover, and canopy height range; however it is possible to define precise crown locations. Scene elements may also be explicitly represented by facets. Spectral reflectance and transmittance properties of the scene elements are also specified, normally approximated as bi-Lambertian. A list of parameters and typical values is given in Table 1. Figure 1 illustrates a typical canopy representation, output from the model under solar illumination conditions.

\subsection{Sensor model}

A generic description of a waveform lidar instrument is defined by parameters giving sensor location, beam energy, beam angular divergence and temporal spread. Both angular divergence and temporal spread are modelled as Gaussian. The set of parameters defining the lidar instrument are given in Table 2, with example values for GLAS used in the current study (Brenner et al., 2000).

\subsection{Evaluation of lidar waveform}

The original model (North, 1996) traced photon trajectories forwards from the source until absorption in the canopy or leaving the canopy boundary, when energy was accumulated in bins defined for each solid angle of exit. Subsequently the model was developed to sample paths from a given view direction to intercepted surfaces, and to accumulate the radiance contribution from these surfaces (Disney et al., 2000; Barton and North, 2002). The latter method is more 
Table 2: Lidar sensor model

\begin{tabular}{cccc}
\hline Name & Units & Meaning & Value \\
\hline$\left(P_{x}, P_{y}, P_{z}\right)$ & $\mathrm{m}$ & Sensor position relative to & $(0,0,600000)$ \\
$\theta_{O}$, & $\mathrm{deg}$ & Sensor zenith angle & 0 \\
$\phi_{O}$ & $\mathrm{deg}$ & Sensor azimuth angle & 0 \\
$\mathrm{sl}$ & $\mathrm{ns}$ & $\begin{array}{c}\text { Emitted RMS pulse width, } \\
\text { assuming Gaussian }(1 \mathrm{sd})\end{array}$ & 5 \\
$q_{T}$ & $\mathrm{rad}$ & Half-width angle of beam & \\
$I F O V$ & $\mathrm{rad}$ & divergence, Gaussian $(1 \mathrm{sd})$ & 0.00011 \\
$A_{T}$ & $\mathrm{~m}$ & Detector IFOV & 0.0004 \\
$T_{R T s t m}$ & - & Detector telescope area & 0.709 \\
$E_{\text {trans }}$ & $\mathrm{mJ}$ & Roundtrip atmospheric trans. & $0.8(532 \mathrm{~nm}) 0.9(1024 \mathrm{~nm})$ \\
$\Delta_{t}$ & $\mathrm{~ns}$ & Total pulse energy & $32(532 \mathrm{~nm}) ; 72(1064 \mathrm{~nm})$ \\
& & Recording bin width & 1 \\
\hline
\end{tabular}

appropriate for lidar calculation, as it is possible to efficiently estimate return for infinitesimal angles; this is necessary for lidar as viewing is made at the retro-reflection direction or 'hot-spot', where the reflectance changes very significantly with small changes in view angle.

The method proceeds by sampling $n$ rays over the instrument IFOV. For each ray:

(i) Find the intersection with the first surface facet (leaf/bark/soil)

(ii) The facet illumination is calculated as the sum of direct and diffuse incoming light. The diffuse light term is calculated by recursive sampling of higher scattering orders. The radiance contribution is defined according to the standard rendering equation, depending on facet orientation with respect to illumination, and optical properties.

(iii) For each facet and scattering order, both the radiance contribution and the total return path length to the sensor are calculated. The path length is equivalent to time of signal. For efficiency, ground-leaving radiance for unit incoming signal is initially recorded.

(iv) The radiance is binned into $m$ bins according to path length, whose width is defined by the sensor model temporal sampling.

The final step accounts for detector characteristics and pulse width:

(v) The radiance values are converted into absolute power $(\mathrm{mW})$ recorded in each temporal bin, dependent on the sensor aperture $A_{T}$, distance to sensor $P_{z}$ and atmospheric round-trip transmission $T_{R T_{s t m}}$, The effect of pulse width is modelled by Gaussian convolution of the resultant output array, with amplitude dependent on emitted pulse energy $E_{\text {trans }}$.

The estimation error decreases as $n^{0.5}$. For results here we use a sampling of $n=10^{4}$, and obtain convergence after scattering order 8. 


\section{Results}

\subsection{Comparison with FLIGHT reflectance}

The waveform was integrated over time and normalised by downwelling radiance to allow comparison with equivalent bidirectional reflectance factor (BRF) for the scenes generated by the original FLIGHT model. While some of the code is common between the original FLIGHT code and the lidar waveform model, the check is useful as the FLIGHT model BRF has been previously checked by intercomparison with other three-dimensional codes as part of the RAdiation Model Intercomparison (RAMI) project (Widlowski et al., 2007). The recent analysis within RAMI of six selected three-dimensional models showed dispersion within $1 \%$ over a large range of canopy descriptions.

The surface reflectance is estimate from the lidar return as:

$$
\rho_{\text {surf }}=\frac{\pi E_{\text {rec }} R^{2}}{E_{\text {trans }} A_{t} T_{\text {RTatm }}}
$$

where $\mathrm{E}_{\mathrm{rec}}(p J)$ is calculated as

$$
E_{\text {rec }}=\sum_{i=1}^{m} L_{i} \Delta_{t}
$$

where energy is accumulated in $m$ sample bins, where each sample bin $i$ has accumulated power $L_{i}(m W)$, and the bins correspond to temporal increment $\Delta_{t} n s$.

Seventeen scenes were generated through independent variation of model parameters described in Table 3, with random spatial positioning of 200 crowns at $70 \%$ fractional cover. Figures 2 and 3 show comparison at $532 \mathrm{~nm}$ and $1064 \mathrm{~nm}$ respectively. Error bars denote scene spatial variation at the scale of the lidar footprint. The results show unbiased estimate of reflectance by the waveform integration, though with scatter about the line. This is expected as the lidar spatial sampling is much smaller than the whole scene simulated by FLIGHT.

\subsection{Sensitivity analysis}

Figures 3-4 show example model runs and sensitivity to variation in plant area index (PAI) and ground slope (S). Table 3 shows full results of a sensitivity analysis of modelled output to variation in canopy parameters. Each parameter is varied individually from a 'base case', specified by the central value in each set in the table; the remaining parameters are specified in Tables 1-2. The lidar waveform return power is recorded, integrated over time and partitioned into total return (TR), canopy return (CR) and ground return (GR). Partition is estimated on the basis of position in the waveform. Variation is shown as a percentage deviation from the base case waveform returns.

- Sensitivity to PAI is small in total return, with less than $2 \%$ variation for PAI from 2-5, from a base case PAI of 3, and a $17 \%$ reduction for PAI of 1 . However the partitioned returns show much greater variation, with $\mathrm{CR}$ decreasing by over $50 \%$, and a corresponding increase in GR over the same range.

- Variation in leaf angle distribution (LAD) shows impact on total return $(-11 \%$ to $+20 \%)$, with a greater impact on CR. Leaf angle will affect both total interception by the canopy by varying projected leaf area, and also the orientation of surfaces with respect to the incoming beam.

- Crown shape has a small impact on total return, with higher vertical and horizontal eccentricities decreasing the CR component relative to spherical crown return. 


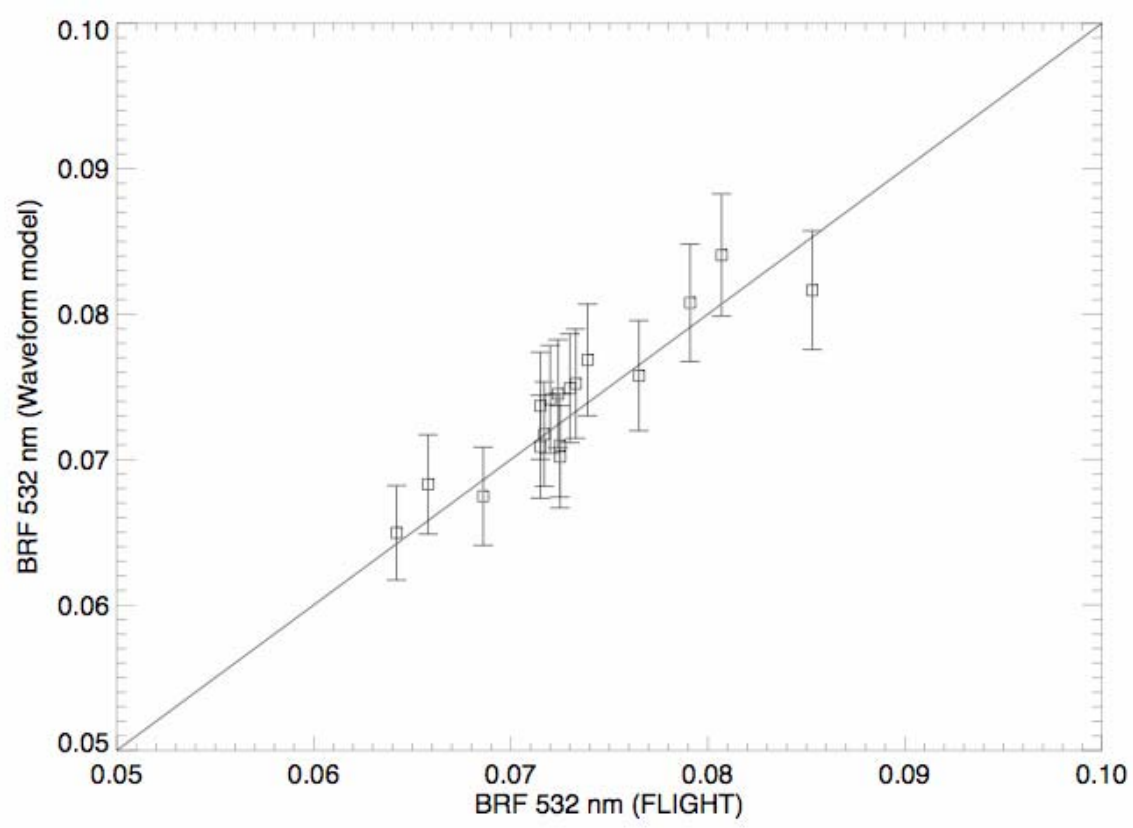

Figure 2: Bidirectional Reflectance Factor (BRF) simulated by FLIGHT vs BRF from time integration of modelled lidar waveform return at $532 \mathrm{~nm}$.

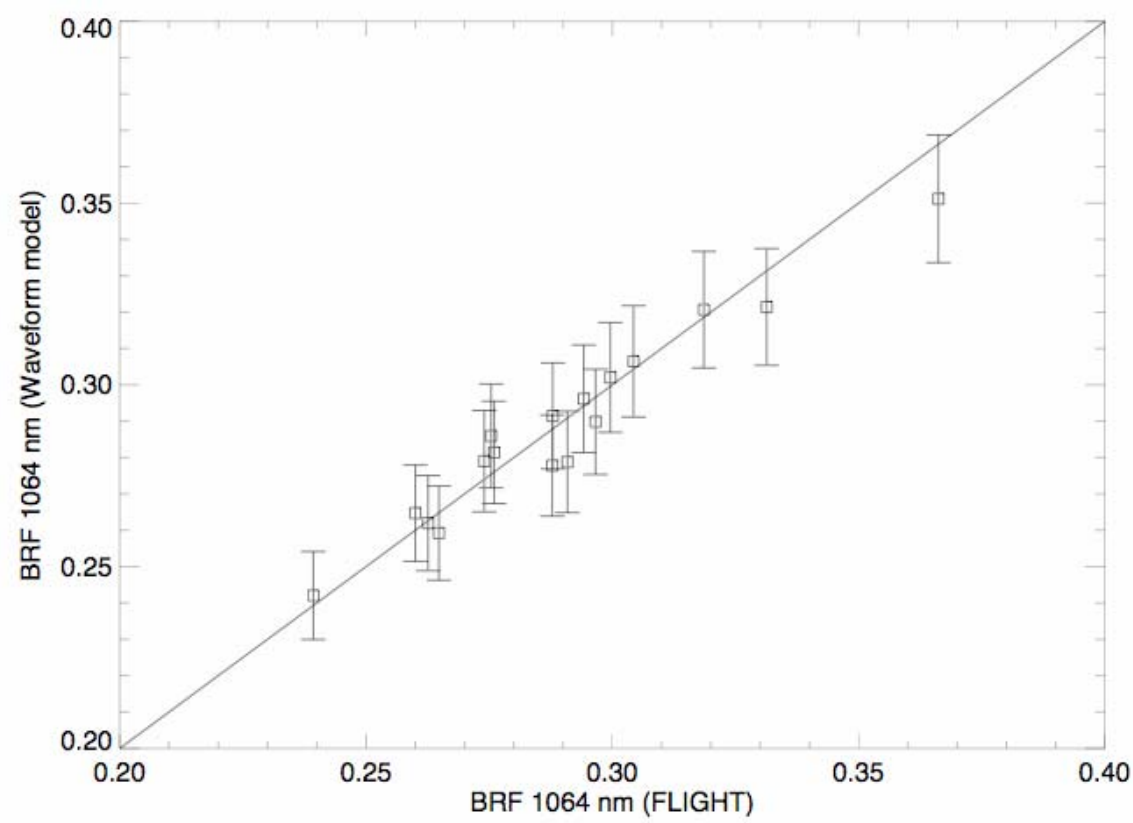

Figure 3: BRF simulated by FLIGHT vs BRF from time integration of modelled lidar waveform return at $1064 \mathrm{~nm}$. 


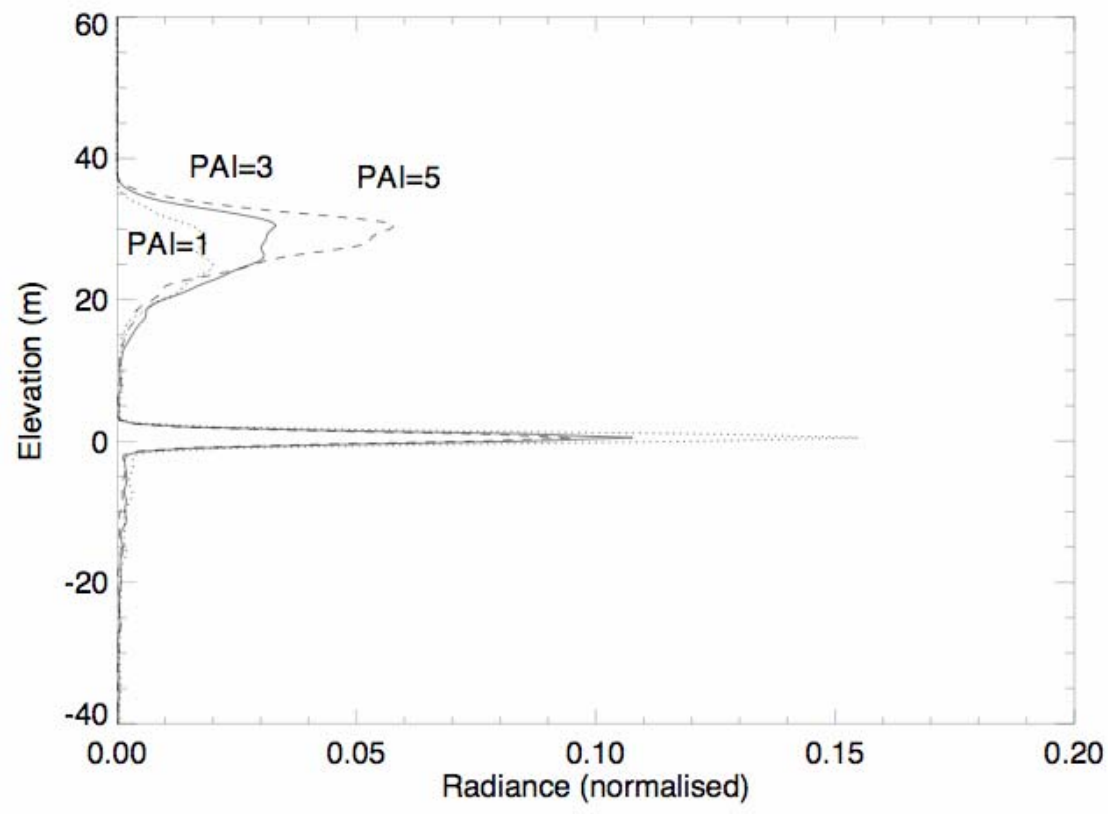

Figure 4: Example model output showing sensitivity of waveform return to plant area index (PAI) variation from 1 to 5 .

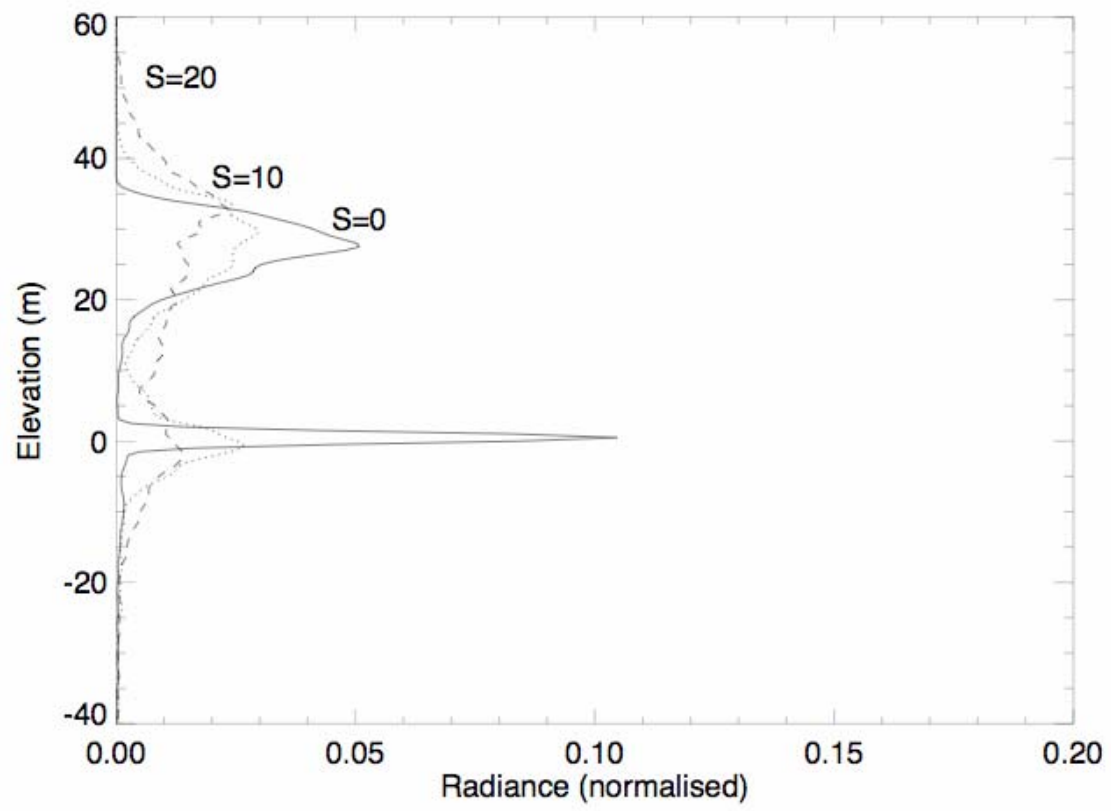

Figure 5: Example model output showing waveform sensitivity to variation in terrain slope, from 0 to 20 degrees. 
Table 3: Sensitivity of waveform lidar return at $1064 \mathrm{~nm}$ to variation in canopy parameters. Return is partitioned into total energy return (TR), canopy return (CR) and ground return (GR). Variation is shown as percentage deviation from the base case return.

\begin{tabular}{|c|c|c|c|c|}
\hline Parameter & Value & TR (\%) & CR (\%) & GR (\%) \\
\hline \multirow{5}{*}{ PAI } & 1 & -17 & -51 & +54 \\
\hline & 2 & -1.8 & -14 & +25 \\
\hline & 3 & - & - & - \\
\hline & 4 & +1.6 & +5.1 & -6.0 \\
\hline & 5 & 0 & +5.2 & -13 \\
\hline \multirow{3}{*}{ LAD } & planophile & +20 & +33 & -5.4 \\
\hline & spherical & - & - & - \\
\hline & erectophile & -11 & -19 & +4.5 \\
\hline \multirow{3}{*}{$E_{z} / E_{x y}$} & .5 & -4.3 & -6.2 & 0 \\
\hline & 1 & - & - & - \\
\hline & 2 & -4.7 & -7.8 & +1.8 \\
\hline \multirow{3}{*}{$\rho_{\mathrm{L}}$} & .45 & +10 & +13 & +2.7 \\
\hline & .4 & - & - & - \\
\hline & .35 & -9.2 & -13 & -2.0 \\
\hline \multirow{3}{*}{$\tau_{\mathrm{L}}$} & .45 & +5.1 & +5.8 & +3.7 \\
\hline & .4 & - & - & - \\
\hline & .35 & -4.3 & -4.9 & -3.0 \\
\hline \multirow{3}{*}{$\rho_{\mathrm{S}}$} & .2 & +10 & 0 & +32 \\
\hline & .15 & - & - & - \\
\hline & .1 & -10 & 0 & -31 \\
\hline
\end{tabular}

Analysis of the leaf optical properties shows sensitivity to canopy reflectance (RL),

- $\quad$ with a slightly greater relative change in CR (13\%) compared to input parameter $(12 \%)$. Sensitivity to multiple scattering is illustrated by the effect of increasing leaf transmittance, and by the increase in GR.

- Variation in soil boundary reflectance (RS) shows direct sensitivity of GR, with impact on total return corresponding to area fraction in scene.

\subsection{Evaluation of indices for canopy parameter retrieval}

While total absolute lidar return is relatively insensitive to vegetation cover, relative to the various perturbing parameters, the differential response of vegetation and ground components suggests use of metrics based on these. However, while reliable separation of a canopy and ground component is frequently problematic, for example in steeply sloping terrain or very 
dense canopies, there are some instances where separate canopy and ground waveforms can be identified. For example Rosette et al. (submitted) explored use of ratio of canopy to total waveform area in correlation with canopy cover in a mixed temperate forest, where separation of ground from canopy was based on Gaussian decomposition of the return pulse. Figure shows an example of a normalised index regressed against the vertically projected plant area index (VPAI). The waveform index (WI) is defined as

$$
W I=(C R-G R) /(C R+G R)
$$

The figure shows the index is sensitive to VPAI $\left(\mathrm{R}^{2}=0.87\right)$ while relatively insensitive to perturbing factors such as variation in leaf and soil optical properties. Such a normalised index would also be insensitive to absolute calibration of the return radiance.

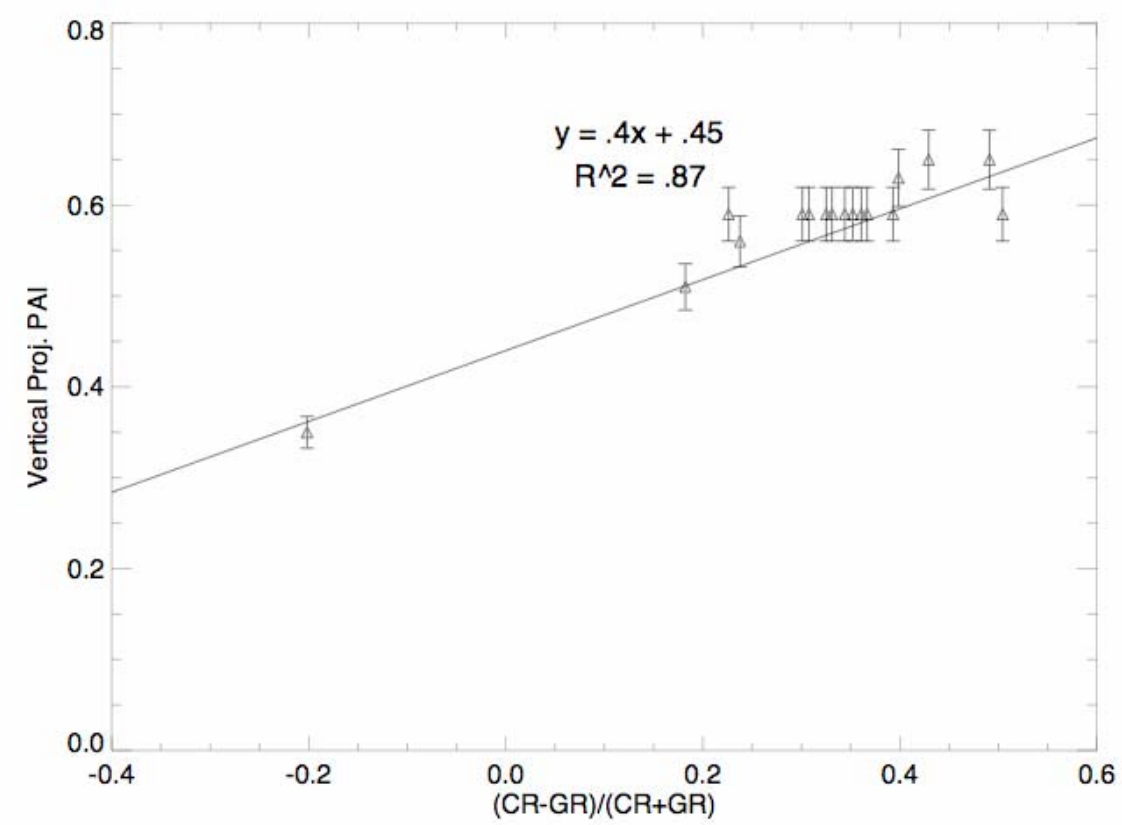

Figure 6: Vertically projected plant area index (PAI) vs index derived from waveform lidar, separated into canopy return $(\mathrm{CR})$ and ground return $(\mathrm{GR})$.

\section{Discussion}

We have presented a Monte Carlo radiative transfer model of waveform lidar for three-dimensional vegetation canopies, within the framework of the FLIGHT model (North 1996). Good agreement is found between the integrated waveform energy and directly derived BRFs from FLIGHT. A sensitivity analysis shows information content in the waveform signal related to canopy cover variation and perturbing factors such as plant area index (PAI) and optical properties. Further research is recommended to accurately model atmospheric scattering and absorption, and to test the model against a wider range of canopies.

\section{Acknowledgements}

This work is supported by the NERC Climate and Land-Surface Systems Interaction Centre, (CLASSIC). We acknowledge scientific input from Dr Malcolm Watson, BAe Systems. 


\section{References}

Alton, P., Mercado, L. and North, P., 2007. A sensitivity analysis of the land-surface scheme JULES conducted for three forest biomes: Biophysical parameters, model processes, and meteorological driving data. GBC 20, GB1008, doi:10.1029/2005GB002653.

Alton, P.B., North, P., Kaduk, J. and Los, S.O., 2005. Radiative transfer modelling of direct and diffuse sunlight in a Siberian pine forest. J. Geophys. Res.,110(D23): D23209.

Barton, C.V.M. and North, P.R.J., 2001. Remote sensing of canopy light use efficiency using the photochemical reflectance index: model and sensitivity analysis. Remote Sensing of Environment, 78, 164-273.

Brenner, A.C., Zwally, H.J., Bentley, C.R. et al. 2000. GLAS Algorithm Theoretical Basis Document 3.0, NASA GSFC.

Disney, M. I., Lewis, P. and Saich, P., 2006. 3D modelling of forest canopy structure for remote sensing simulations in the optical and microwave domains, Remote Sensing of Environment 100(1), 114-132.

Disney, M.I., Lewis, P. and North, P.R.J., 2000. Monte Carlo ray tracing in optical canopy reflectance modelling. Remote Sensing Reviews, 18( 2-4), 197-226.

Govaerts, Y.M. and Verstraete, M.M., 1998. Raytran: A Monte Carlo ray-tracing model to compute light scattering in three-dimensional heterogeneous media. IEEE Transactions on Geoscience and Remote Sensing, 36(2), 493-505.

Harding, D. J., and Carabajal, C. C., 2005. ICESat waveform measurements of within-footprint topographic relief and vegetation vertical structure. Geophysical Research Letters, 32, L21S10, doi:10.1029/2005GL023471.

Koetz, B., Morsdorf, F., Sun, G. et al., 2006. Inversion of a Lidar Waveform Model for Forest Biophysical Parameter Estimation. IEEE Geoscience and Rem. Sens. Letters, 3(1).

Kotchenova, S.Y., Shabanov, N.V., Knyazikhin, Y., et al., 2003. Modeling lidar waveforms with time-dependent stochastic radiative transfer theory for remote estimations of forest structure. Journal of Geophysical Research, 108(D15), 4484, doi:10.1029/2002JD003288.

Lefsky, M., Harding, D., Keller, M., et al., 2005. Estimates of forest canopy height and aboveground biomass using ICESat. Geophysical Research Letters, 32 (L22S02).

Ni-Meister, W., Jupp, D.L.B., and Dubayah, R., 2001. Modeling lidar waveforms in heterogeneous and discrete canopies. IEEE TGARS 39(9), 1943-1958.

North, P.R.J., 1996. Three-dimensional forest light interaction model using a Monte Carlo method. IEEE Transactions on Geoscience and Remote Sensing 34(5): 946-956.

North, P.R.J., 2002. Estimation of fAPAR, LAI and vegetation fractional cover from ATSR-2 imagery. Remote Sensing of Environment, 80, 114-121.

Platt, C.M.R., 1981. Remote Sounding of High Clouds. III: Monte Carlo Calculations of Multiple-Scattered Lidar Returns. Journal of the Atmospheric Sciences, 38(1): 156-167.

Rosette, J.A., North, P.R.J. and Suárez, J.C., 2008a. Vegetation Height Estimates for a Mixed Temperate Forest using Satellite Laser Altimetry. Int. J. of Rem. Sens., 29(5): 1475-1493.

Rosette, J.A., North, P.R.J. and Suárez, J.C., 2008b. Satellite lidar estimation of stemwood volume: a method using waveform decomposition. Photogramm. J. Finland, 21(1): 76-85.

Rosette, J.A., et al., submitted. A Comparison of Biophysical Parameter Retrieval for Forestry using Airborne and Satellite LiDAR. Int. J. of Remote Sens,.

Sellers, P. J., Los S. O., Tucker C. J., et al., 1996. A revised land surface parameterization (SiB-2) for atmospheric GCMs. Part 2: The generation of global fields of terrestrial biophysical parameters from satellite data. Journal of Climate, 9(4), 706-737.

Schutz B., Zwally, H., Shuman, C., Hancock, D. and DiMarzio, J., 2005. Overview of the ICESat Mission. Geophysical Research Letters 32: L21S01

Sun, G., Ranson, K.J., Masek, J., et al., 2008. Estimation of Tree Height and Forest Biomass from GLAS Data. Journal of Forest Planning, 13: 157-164.

Widlowski, J-L., Taberner, M., Pinty, B. et al., 2007. The third RAdiation transfer Model Intercomparison (RAMI) exercise: Documenting progress in canopy reflectance models. Journal of Geophysical Research, 112, D09111, doi:10.1029/2006JD007821. 